\title{
Impaired toll-like receptor 8 signaling in multiple sclerosis
}

\author{
Tory P Johnson ${ }^{1}$, Richa Tyagi ${ }^{1}$, Karan Patel ${ }^{1}$, Nicoline Schiess ${ }^{2}$, Peter A Calabresi ${ }^{2}$ and Avindra Nath $^{1 *}$
}

\begin{abstract}
Background: The etiology and immunopathology of multiple sclerosis (MS) is not well understood. It is recognized that although autoreactive T cells are the main early mediators of disease, other cell types, including cells of the innate immune system contribute to MS pathogenesis. The objective of this study was to determine if Toll-like receptor (TLR) signaling is functionally altered in patients with MS.

Findings: Peripheral blood mononuclear cells from healthy donors and patients with relapsing remitting MS were stimulated with specific agonists of TLRs 3, 7, 8 and 9. Using quantitative polymerase chain reaction transcript levels of tumor necrosis factor-a, interferon- $a$ and interleukin (IL)-12 $\beta$ were quantified from patients with MS and healthy donors. TLR8-induced production of IL12B transcripts and protein was functionally impaired in patients with MS as compared to healthy controls $(P<0.05$ and $P<0.005$, respectively). Patients with MS also expressed lower baseline levels of TLR8 as compared to healthy controls $(P<0.05)$.
\end{abstract}

Conclusions: TLR8 expression and signaling is impaired in peripheral blood mononuclear cells from patients with MS. This finding suggests that loss of TLR8 signaling may be contributing to autoimmune processes in MS.

Keywords: Toll-like receptor, Multiple sclerosis, Gene expression, Interleukin 12, TLR8

\section{Findings}

Multiple sclerosis (MS) is an autoimmune disorder characterized by demyelination, chronic inflammation, and neuronal damage primarily caused by activated, auto-reactive CD4+ and CD8+ T cells directed against myelin [1]. Combinations of genetic and environmental factors, including pathogens, are believed to trigger the abnormal immune activation in MS [2]. Pathogens which initiate innate immune responses in the central nervous system (CNS) via Toll-like receptor (TLR) signaling may lead to the development of autoreactive $T$ cells due to antigen spread in a process known as bystander activation $[2,3]$.

TLRs are pattern recognition receptors (PRRs) that recognize conserved pathogen-associated molecular patterns (PAMPs) [4]. TLRs responsible for detecting viral PAMPs include TLR3, which detects viral double stranded (ds)RNA, TLR7 and TLR8, which detect viral single stranded (ss)RNA, and TLR9, which detects dsDNA and

\footnotetext{
* Correspondence: avindra.nath@nih.gov

'Section of Infections of the Nervous System, National Institute of Neurological Disorders and Stroke, National Institutes of Health, Bldg 10; 7C103, 10 Center Drive, Bethesda, MD 20892, USA

Full list of author information is available at the end of the article
}

are located in the endosome [5,6]. TLR signaling results in the activation of $\mathrm{T}$ cells [7] and production of proinflammatory cytokines, such as interleukin (IL)-12 $\beta$, interferon (IFN) and tumor necrosis factor (TNF)- $\alpha$, which are known to be increased in patients with MS $[5,8,9]$. Many studies have implicated TLRs in the pathophysiology of MS and experimental autoimmune encephalomyelitis (EAE) [4]. For instance, activation of TLRs reliant on the downstream adaptor protein MyD88 (TLR7, 8 and 9) may enhance MS progression, while the activation of TLR3, which is not dependent on MyD88, may be protective in neurons $[4,10]$. However, it remains unclear if TLR signaling is functionally dysregulated in patients with MS prior to treatment. To better understand the role of TLR signaling in MS, we sought to determine the functional properties of TLRs 3, 7, 8 and 9 in these patients as compared to healthy donors.

To determine if patients with MS had dysregulated TLR signaling we analyzed the transcriptional response of IL12 $\beta$, IFN- $\alpha$ and TNF- $\alpha$ to TLR3, 7, 8 and 9 stimulation. Briefly, peripheral blood mononuclear cells (PBMC) were obtained from clinically stable untreated patients with relapsing remitting MS $(\mathrm{n}=10)$ and from healthy 
age- and gender-matched donors $(\mathrm{n}=10)$. Patients with MS were aged from 49 to 74 years (median 57 years; seven females and three males). Healthy donors ranged in age from 41 to 72 years (median 57.5; seven females and three males). All patients and donors gave informed consent and this study was approved by the Office of Human Subjects Protection and Research at the National Institutes of Health. PBMCs from healthy donors and patients with MS were isolated by a standard operating procedure using Ficoll-Isopaque density gradient centrifugation (Gibco, Life Technologies Ltd., Paisley, UK) and were immediately stored in liquid nitrogen until further use. Cells from controls and patients with MS were processed under the same protocol. Cells were maintained in Iscove's Modified Dulbecco's Media (Invitrogen, Carlsbad, CA, USA) supplemented with $10 \%(\mathrm{v} / \mathrm{v})$ human serum (Sigma, St. Louis, MO, USA) and $1 \%(\mathrm{v} / \mathrm{v})$ antibiotic-antimycotic mixture (Invitrogen) at $37^{\circ} \mathrm{C}$ with $5 \% \mathrm{CO}_{2}$. PBMCs were plated at $1.5 \times 10^{6}$ cells/well and treated with either media (unstimulated), phytohaemagglutinin (PHA) $(0.025 \mu \mathrm{g} / \mathrm{mL})$, TLR3 ligand, Poly(I:C) $(10 \mu \mathrm{g} / \mathrm{mL})$, TLR7 ligand, Imiquimod $(25 \mu \mathrm{g} / \mathrm{mL})$, TLR8 ligand, ssPolyU/LyoVec $(10 \mu \mathrm{g} / \mathrm{mL})$ or TLR9 ligand, ODN2216 $(0.025 \mu \mathrm{g} / \mathrm{mL})$ for six hours at $37^{\circ} \mathrm{C}$. All ligands were obtained from InvivoGen (San Diego, CA, USA). After incubation, cells were collected and total RNA was extracted using the QIAGEN RNeasy Plus kit (Qiagen, Germantown, MD, USA). Up to 100 ng of RNA from each sample was reverse transcribed into cDNA per the manufacturer's instruction using the SuperScript III First-Strand Synthesis SuperMix for quantitative-PCR (Invitrogen). Gene expression levels for IFNA2, TNFA, IL12B and glyceraldehyde 3-phosphotate dehydrogenase $(G A P D H)$ were determined by quantitative PCR performed on a ViiA ${ }^{\text {ma }} 7$ Real-Time PCR System (Applied Biosystem, Carlsbad, CA, USA). All primers and standards were obtained
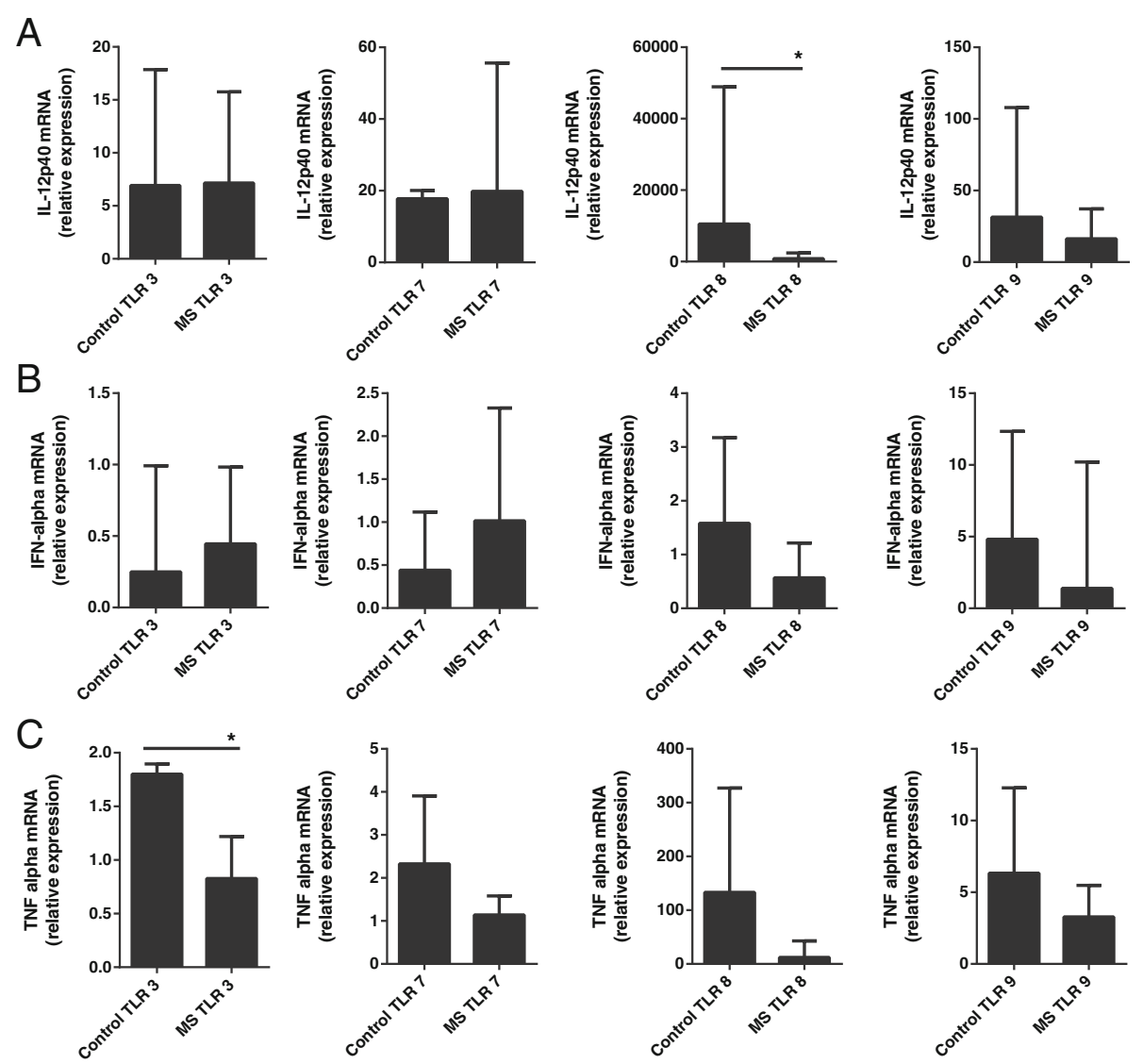

Figure 1 PBMC cytokine gene expression levels after TLR stimulation. (A) IL-12B transcripts were decreased in patients with MS as compared to healthy donors after Toll-like receptor (TLR) 8 stimulation (Student's unpaired two-tailed $t$-test, ${ }^{*}=P<0.05, \mathrm{n}=10$ per group) but not after TLR 3, TLR 7 or TLR 9 stimulation. (B) There was no difference between patients with MS and healthy donors in IFN-alpha transcript production after TLR stimulation. (C) TNF-alpha transcripts were decreased in patients with MS as compared to healthy donors after TLR 3 stimulation (Student's unpaired two-tailed $t$-test, ${ }^{*}=P<0.05, \mathrm{n}=10$ per group) but not after TLR 7, TLR 8 or TLR 9 stimulation. Data shown are median $\pm 25 \%$ to $75 \%$ interquartile range relative gene expression of each cytokine normalized to GAPDH and a reference sample. PBMC, peripheral blood mononuclear cells. 
from Origene (Origene, Rockville, MD, USA). Cytokine transcript expression levels were determined by relative quantification ( $\Delta \Delta \mathrm{Ct}$ method [11]) for each individual using GAPDH for normalization. Cells from patients with MS showed a 58-fold decrease in transcript levels of $I L 12 B$ in response to TLR8 stimulation (median \pm $25 \%$ to $75 \%$ interquartile range $=179.3 \pm \pm 56.0$ to 896.0 versus $10,518 \pm 1,607$ to 48,932 relative expression; $P<0.05)$ (Figure 1A), but no difference was detected after TLR3, TLR7 or TLR9 stimulation. No significant difference was found between patients with MS and healthy donors in production of IFNA with any TLR stimulation (Figure 1B). Patients with MS showed a two-fold decrease in transcript levels of TNFA in response to TLR3 stimulation (median $\pm 25 \%$ to $75 \%$ interquartile range $=0.9 \pm 0.4$ to 1.0 versus $1.8 \pm 1.1$ to 1.9 relative expression; $P<0.05$ ) as compared to healthy donors (Figure $1 \mathrm{C}$ ), but not after TLR7, 8 or 9 stimulation.

As MS is a hyper-inflammatory disorder and as IL12 is known to be elevated in patients with MS [12], we were surprised to see a decrease in $I L 12 B$ production from patients with MS. We, therefore, confirmed these results by determining the concentration of IL$12 \mathrm{p} 40$ produced after TLR8 stimulation. Briefly, $1.5 \times$ $10^{6}$ cells were plated and TLR8 was stimulated as above for 24 hours. After incubation, supernatants were collected and analyzed for IL12p40 protein secretion by enzyme-linked immunosorbent assay (ELISA) (Cell Sciences, Canton, MA, USA). In accordance with the transcription data, we found a decrease in IL12p40 protein secreted from PBMCs from patients with MS as compared to healthy controls after TLR8 stimulation (mean $\pm \mathrm{SE}=717.7 \pm 211.3$ versus $2,401 \pm 285.4 \mathrm{pg} / \mathrm{ml}$; $P<0.005$ ) but not after PHA stimulation (mean \pm SE $=$ $843.5 \pm 287.6$ versus $709.4 \pm 169.8 \mathrm{pg} / \mathrm{ml} ; P>0.05)$ (Figure 2) indicating the cells from patients with MS were capable of responding to the same level as cells from healthy donors. No appreciable production of IL12p40 was observed after stimulation with TLR3, 7 or 9 (Figure 2).

We next analyzed the baseline expression of TLR8 and TLR3 in patients with MS as well as healthy donors. Using RNA harvested from unstimulated cells as described above, quantitative PCR was performed using primers specific for TLR8 or TLR3 and GAPDH. TLR transcript expression levels were determined by relative quantification as described above. Cells from patients with MS showed a 2.7fold decrease in transcript levels of TLR8 (median $\pm 25 \%$ to $75 \%$ interquartile range $=0.3 \pm 0.1$ to 0.6 versus $0.8 \pm$ 0.6 to 1.2 relative expression; $P<0.005)$ (Figure $3 \mathrm{~A}$ ); no difference in TLR3 expression was detected (median $\pm 25 \%$ to $75 \%$ interquartile range $=2.8 \pm 1.5$ to 3.7 versus $2.7 \pm$ 1.7 to 4.0 relative expression; $P>0.05$ ) as compared to healthy donors (Figure 3B).

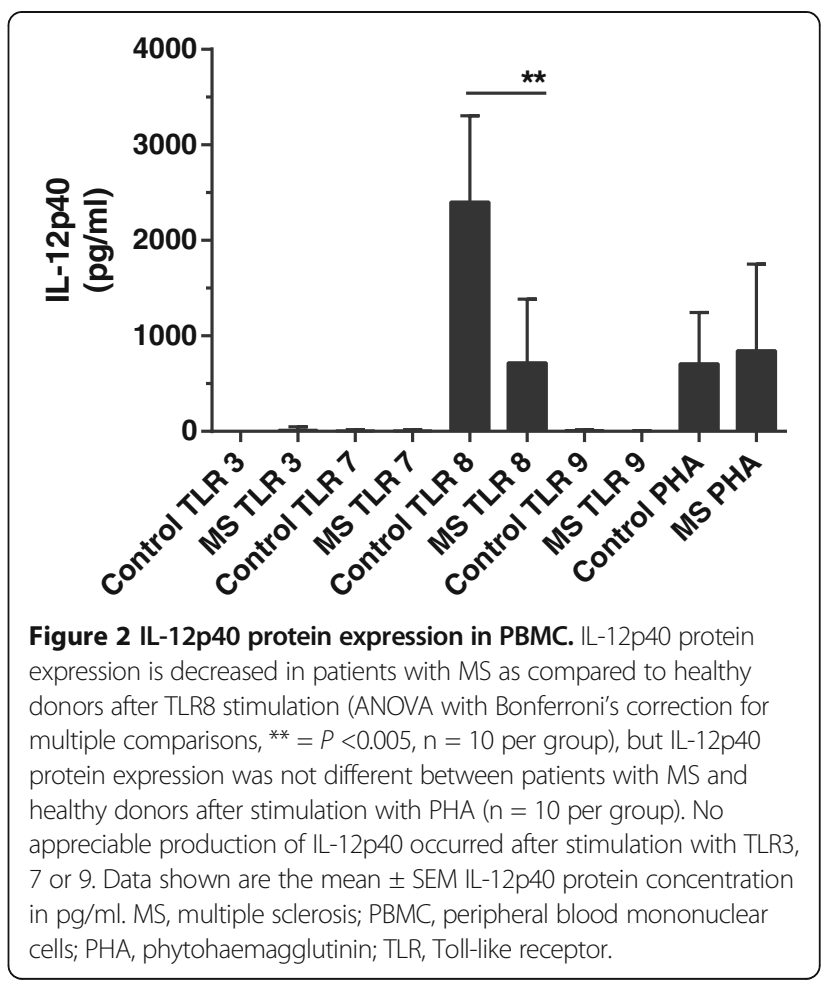

\section{Conclusions}

This study shows that patients with MS exhibit a profound dysregulation of TLR8 signaling as compared to healthy donors manifested by IL- $12 \beta$ transcript and IL$12 \mathrm{p} 40$ protein production. The mechanism of depressed responsiveness of TLR8 appears to be a result of decreased TLR8 baseline expression in PBMC from patients with MS. Our data indicate that TLR8 signaling in

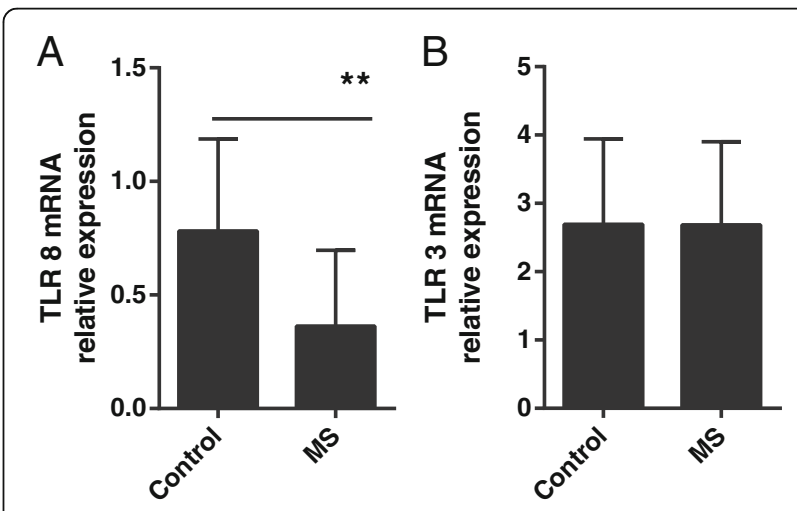

Figure 3 TLR 8 and 3 gene expression in PBMC. Patients with MS and healthy donors differ in baseline (A) TLR8 transcript expression (Student's unpaired two-tailed $t$-test, ${ }^{* *}=P<0.005, \mathrm{n}=10$ per group) but not in (B) TLR3 transcript expression ( $n=7$ per group). Data shown are median $\pm 25 \%$ to $75 \%$ interquartile range relative TLR mRNA quantification at baseline for each individual normalized to GAPDH and a reference sample. MS, multiple sclerosis; PBMC, peripheral blood mononuclear cells; TLR, Toll-like receptor. 
patients with MS may be functionally hypoactive, resulting in detectable under-production of IL-12 $\beta$. IL12B encodes the p40 subunit of IL-12 which forms both IL-12 and IL23, two cytokines that are considered a driving force in EAE progression [13-16]. Although IL12p40 appears to have a critical role in EAE, MS pathogenesis may not be dependent on IL-12p40. A clinical trial using ustekinumab, a monoclonal antibody against IL-12p40, in patients with MS showed no beneficial clinical effect [16]. Therefore, the lack of IL12p40 detected in our study does not conflict with current data, although it seems counter-intuitive in an auto-immune mediated disease. Our study also detected a statistical difference in TNF $\alpha$ production after TLR3 stimulation; however, the expression values were low and the difference was rather modest, hence its biological relevance is unknown. We looked specifically at functionality of a subset of TLRs with a limited cytokine output. Our study is unique because we studied untreated patients in the absence of a clinical relapse. We readily detected a robust difference in TLR8 function between patients with MS and healthy donors. This finding may be clinically relevant as TLR8 deficient mice have increased autoimmunity [17]. Interplay between TLR8 loss and hyper-expression of TLR7 has been documented in mice [17] and patients with MS who are treated with INF- $\beta 1$ therapy have an increase in TLR7 expression [18]. TLR8 is the least well understood of the human TLRs and our study provides additional evidence that TLR8 needs further investigation and, similar to the murine model, that human TLR8 deficiency may contribute to autoimmune processes.

\section{Abbreviations \\ CNS: Central nervous system; Ds: Double stranded; EAE: Experimental autoimmune encephalomyelitis; ELISA: Enzyme-linked immunosorbent assay; GAPDH: Glyceraldehyde 3-phosphotate dehydrogenase; IFN: Interferon; IL: Interleukin; MS: Multiple sclerosis; PAMP: Pathogen-associated molecular patterns; PBMC: Peripheral blood mononuclear cells; \\ PHA: Phytohaemagglutinin; PRR: Pattern recognition receptors; Ss: Single stranded; TLR: Toll-like receptor; TNF: Tumor necrosis factor.}

\section{Competing interests}

All authors declare that they have no competing interests.

\section{Authors' contributions}

TPJ designed, performed and analyzed the experiments, coordinated collaborations and wrote the manuscript. RT and KP performed and analyzed experiments. NS designed the experiments and conducted the pilot studies. PAC assisted in experimental design. AN conceived of the project, designed and analyzed the experiments, coordinated collaborations and wrote the manuscript. All authors read and approved the final manuscript.

\section{Acknowledgments}

The authors would like to acknowledge Dr. Steve Jacobson for providing the clinical samples. This work was supported by the National Multiple Sclerosis Society (grant number TR 3760-A-3); a generous grant from the Stegg family; and NINDS intramural funds.

\section{Author details}

${ }^{1}$ Section of Infections of the Nervous System, National Institute of Neurological Disorders and Stroke, National Institutes of Health, Bldg 10; 7C103, 10 Center Drive, Bethesda, MD 20892, USA. ²Department of Neurology, Johns Hopkins University, Baltimore, MD, USA.
Received: 14 April 2013 Accepted: 14 June 2013

Published: 21 June 2013

\section{References}

1. Friese MA, Fugger L: Pathogenic CD8(+) T cells in multiple sclerosis. Ann Neurol 2009, 66:132-141.

2. Racke MK, Drew PD: Toll-like receptors in multiple sclerosis. Curr Top Microbiol Immunol 2009, 336:155-168.

3. McMahon EJ, Bailey SL, Castenada CV, Waldner H, Miller SD: Epitope spreading initiates in the CNS in two mouse models of multiple sclerosis. Nat Med 2005, 11:335-339.

4. Gambuzza M, Licata N, Palella E, Celi D, Foti Cuzzola V, Italiano D, Marino S, Bramanti P: Targeting Toll-like receptors: emerging therapeutics for multiple sclerosis management. J Neuroimmunol 2011, 239:1-12.

5. Blasius AL, Beutler B: Intracellular toll-like receptors. Immunity 2010, 32:305-315.

6. Okun E, Griffioen KJ, Mattson MP: Toll-like receptor signaling in neural plasticity and disease. Trends Neurosci 2011, 34:269-281.

7. Funderburg N, Luciano AA, Jiang W, Rodriguez B, Sieg SF, Lederman MM: Toll-like receptor ligands induce human T cell activation and death, a model for HIV pathogenesis. PLoS One 2008, 3:e1915.

8. Ensoli F, Fiorelli V, Lugaresi A, Farina D, De Cristofaro M, Collacchi B, Muratori DS, Scala E, Di Gioacchino M, Paganelli R, Aiuti F: Lymphomononuclear cells from multiple sclerosis patients spontaneously produce high levels of oncostatin $\mathrm{M}$, tumor necrosis factors alpha and beta, and interferon gamma. Mult Scler 2002, 8:284-288.

9. Obradovic D, Kataranovski M, Dincic E, Obradovic S, Colic M: Tumor necrosis factor-alfa and interleukin-4 in cerbrospinal fluid and plasma in different clinical forms of multiple sclerosis. Vojnosanit Pregl 2012, 69:151-156.

10. Fischer M, Ehlers M: Toll-like receptors in autoimmunity. Ann N Y Acad Sci 2008, 1143:21-34.

11. Livak KJ, Schmittgen TD: Analysis of relative gene expression data using real-time quantitative PCR and the 2(-Delta Delta $C(T)$ ) method. Methods 2001, 25:402-408.

12. Kouwenhoven M, Teleshova N, Ozenci V, Press R, Link H: Monocytes in multiple sclerosis: phenotype and cytokine profile. J Neuroimmunol 2001, 112:197-205.

13. Unutmaz D, Pileri P, Abrignani S: Antigen-independent activation of naive and memory resting T cells by a cytokine combination. J Exp Med 1994, 180:1159-1164.

14. Langrish CL, Chen Y, Blumenschein WM, Mattson J, Basham B, Sedgwick JD, McClanahan T, Kastelein RA, Cua DJ: IL-23 drives a pathogenic T cell population that induces autoimmune inflammation. J Exp Med 2005, 201:233-240.

15. Segal BM, Shevach EM: IL-12 unmasks latent autoimmune disease in resistant mice. J Exp Med 1996, 184:771-775.

16. Constantinescu CS, Wysocka M, Hilliard B, Ventura ES, Lavi E, Trinchieri G, Rostami A: Antibodies against IL-12 prevent superantigen-induced and spontaneous relapses of experimental autoimmune encephalomyelitis. J Immunol 1998, 161:5097-5104.

17. Demaria O, Pagni PP, Traub S, de Gassart A, Branzk N, Murphy AJ, Valenzuela DM, Yancopoulos GD, Flavell RA, Alexopoulou L: TLR8 deficiency leads to autoimmunity in mice. J Clin Invest 2010, 120:3651-3662.

18. Zhang X, Jin J, Tang Y, Speer D, Sujkowska D, Markovic-Plese S: IFN-beta1a inhibits the secretion of Th17-polarizing cytokines in human dendritic cells via TLR7 up-regulation. J Immunol 2009, 182:3928-3936.

doi:10.1186/1742-2094-10-74

Cite this article as: Johnson et al:: Impaired toll-like receptor 8 signaling in multiple sclerosis. Journal of Neuroinflammation 2013 10:74. 